\title{
Alterações orofaciais em doenças alérgicas de vias aéreas
}

\author{
Orofacial alterations in allergic diseases of the airways
}

Anete Branco ${ }^{1}$, Giesela Fleischer Ferrari², Silke Anna T. Weber ${ }^{3}$

\section{RESUMO}

Objetivo: Apontar as possíveis alterações orofaciais decorrentes do sintoma "obstrução nasal" em pacientes portadores de doenças alérgicas de vias aéreas superiores, por meio de revisão de literatura.

Fontes de dados: Levantamento bibliográfico utilizando bancos de dados eletrônicos, como Medline, Ovid, SciELO e Lilacs, com as palavras-chave "asthma", "rhinitis" e "mouth breathing", abrangendo os 30 últimos anos. Foram incluídos artigos de revisão, estudos observacionais e ensaios clínicos.

Síntese dos dados: A obstrução nasal é encontrada freqüentemente em doenças alérgicas de vias aéreas, como rinite e asma. A respiração bucal decorrente da obstrução nasal pode interferir de maneira direta no desenvolvimento infantil, com alterações no crescimento do crânio e orofacial, na fala, na alimentação, na postura corporal, na qualidade do sono e no desempenho escolar.

Conclusões: Devido à variedade de alterações orofaciais encontradas na criança respiradora bucal decorrente de obstrução nasal por doenças alérgicas de vias aéreas, é necessário realizar diagnóstico e tratamento precoces por uma equipe multidisciplinar, composta por médico, ortodontista e fonoaudiólogo, contemplando a visão de uma via respiratória única, que traz consequiências ao crescimento e desenvolvimento do sistema motor oral.

Palavras-chave: desenvolvimento maxilofacial; hipersensibilidade; doenças respiratórias; respiração bucal; rinite alérgica perene.

\section{ABSTRACT}

Objective: To study possible orofacial alterations originated from nasal obstruction symptoms in patients with allergic diseases of the superior airways, through search of scientific literature about the theme.

Data sources: Bibliographic survey of the last 30 years using electronic data such as Medline, Ovid, SciELO and Lilacs, and the keywords "asthma", "rhinitis" and "mouth breathing". Revision articles, observational and clinical studies were included.

Data synthesis: Nasal obstruction is often found in patients with allergic diseases of airways, such as rhinitis and asthma. The mouth breathing originated from nasal obstruction can interfere directly in the child development with alterations in the craniofacial growth, body posture, sleep quality and performance at school.

Conclusions: Due to the variety of orofacial alterations found in children who have nasal obstruction because of allergic diseases of airways and, therefore, are mouth breathers, it is necessary to start the treatment as soon as possible by a multidisciplinary team, formed by doctors, orthodontists and speech therapists, considering a unique airway that brings consequences to growth and development of the oral motor system of these children.

Key-words: maxillofacial development; hipersensitivity; respiratory tract diseases; mouth breathing; rhinitis, allergic, perennial.

\footnotetext{
${ }^{1}$ Mestre em Pediatria da Faculdade de Medicina de Botucatu da Universidade Estadual Paulista (Unesp)

${ }^{2}$ Professora assistente doutora do Departamento de Pediatria da Faculdade de Medicina de Botucatu da Unesp

${ }^{3}$ Professora assistente doutora do Departamento de Otorrinolaringologia e Oftalmologia da Faculdade de Medicina de Botucatu da Unesp
}

Endereço para correspondência:

Anete Branco

Rua João Marques, 82

CEP 18530-000 - Tietê/SP

E-mail: anete.branco@uol.com.br

Recebido em: 12/3/2007

Aprovado em: 10/7/2007 


\section{Introdução}

Embora subjetiva, a obstrução nasal gera uma sensação de desconforto ocasionada pela insuficiente passagem de ar pelo nariz ${ }^{(1)}$, não indicando necessariamente a presença de um obstáculo anatômico na corrente aérea, como, por exemplo, desvio de septo, hipertrofia de cornetos, adenóides e de amígdalas palatinas, tumores e pólipos, mas, com freqüência, indica a presença de processo inflamatório, como ocorre nas rinites alérgicas e asma.

Encontra-se prevalência de até 98,9\% de rinite em asmáticos com atopia e de até 78,4\% em asmáticos sem atopia ${ }^{(2)}$, não havendo boa definição na literatura da prevalência de alterações orofaciais na rinite ou no respirador oral sem rinite. A rinite é um marcador de gravidade para a asma. A combinação e a interação entre ambas acontecem de modo variável, modificando-se segundo o indivíduo, a predisposição genética, a estação do ano, a exposição aos alérgenos e a idade do paciente ${ }^{(3)}$. Annesi-Maesano ${ }^{(4)}$ descreve alguns determinantes bem como fatores de risco em relação às inter-relações da asma e rinite: fatores genéticos, história familiar de alergia, atopia, fatores imunológicos, tabagismo passivo e ativo e poluição; entretanto, hiper-responsividade brônquica, ar frio, umidade, variações sazonais, nutrição, hábitos de vida, condições socioeconômicas e infecções podem ou não estar evidentes. Embora o mecanismo imunológico seja semelhante entre rinite e asma ${ }^{(5)}$, há diferenças histopatológicas na mucosa nasal e brônquica. Observa-se, na cavidade nasal, um sistema de vasos que, durante a congestão, expande a mucosa e invade a luz aérea, favorecendo a obstrução. Tais vasos estão ausentes ou menos exuberantes na árvore traqueobrônquica. Estas diferenças na estrutura e função da mucosa nasal e das vias aéreas estão associadas ao papel do nariz de filtrar e condicionar o ar inspirado, controlar a temperatura corporal e o conteúdo de água ${ }^{(6)}$.

A literatura evidencia que a relação mais importante entre asma e rinite é a presença de inflamação nas mucosas nasal e da via aérea inferior ${ }^{(7)}$, decorrente do contato com o antígeno na via aérea superior, o que provoca, portanto, inflamação em toda a via aérea ${ }^{(8)}$. Na rinite alérgica, o epitélio pode ser preservado ou há descamação sem espessamento da membrana basal, diferente do observado na asma ${ }^{(9)}$.

A relação entre rinite e asma não está claramente estabelecida: podem se tratar de duas entidades distintas ou de uma doença que envolve ambas as vias aéreas. A rinite alérgica é fator de risco para o desenvolvimento da asma ${ }^{(5,7,9-11)}$, ou seja, a maioria dos pacientes com asma tem rinite.
$\mathrm{Na}$ crise de rinite alérgica, há espirros, rinorréia, sintomas oculares e obstrução nasal; já na asma, há episódios de tosse, dispnéia, respiração curta, pulmão com ruído em combinação com broncoconstrição e hiper-reatividade ${ }^{(12)}$.

Encontram-se, na obstrução nasal, deficiências no aquecimento e umidificação do ar inspirado e na própria função filtrante nasal, sendo que a respiração oral resultante permite que os aeroalérgenos atinjam as vias aéreas inferiores, provocando a hiper-reatividade brônquica e a asma induzida pelo exercício ${ }^{(9)}$. No estudo de Kanani et a $l^{(11)}$, a gravidade da asma em pacientes asmáticos atópicos foi menor naqueles com sintomas nasais e, de modo inverso, nos pacientes asmáticos não-atópicos, a asma foi mais grave naqueles que não tinham sintomas nasais.

Informações obtidas com o próprio paciente sobre o grau de obstrução, se esta é uni ou bilateral, contínua ou se ocorre em períodos ou situações específicos ${ }^{(1)}$, se está associadas a outras queixas, além dos achados em exames, direcionam o clínico a um diagnóstico preciso. Lembrando que a respiração é uma das funções vitais do organismo humano, seu desequilíbrio causa inúmeras alterações em vários órgãos e sistemas.

\section{Respiração oral e desenvolvimento craniofacial}

A respiração nasal assume um papel protetor das cavidades paranasais, auriculares e das vias aéreas inferiores ${ }^{(13)}$, não podendo ser separada do restante do trato respiratório, uma vez que sua função primordial é o preparo do ar para que haja melhor aproveitamento deste nos pulmões ${ }^{(14)}$. Além disso, a respiração nasal é fundamental para o crescimento e desenvolvimento adequados do complexo craniofacial e para o funcionamento das funções estomatognáticas do indivíduo ${ }^{(15)}$. A função respiratória normal se faz por via nasal desde o nascimento e assim deve ser pelo resto da vida, mesmo com a maior resistência à passagem de ar inalado pela via aérea nasal ${ }^{(16)}$.

Quando há obstrução nasal, o padrão respiratório é alterado em maior ou menor grau, favorecendo a respiração oral. Existem casos em que a respiração oral é apenas um hábito e as vias aéreas superiores estão desobstruídas, mas a criança continua com a respiração viciosa ${ }^{(17)}$.

O efeito da obstrução nasal sobre o crescimento facial e dental é bastante controverso, em função do critério muitas vezes subjetivo utilizado para definir a respiração bucal. A falta de objetividade dos exames pode levar a diagnóstico incorreto e, conseqüentemente, a tratamento não apropriado ${ }^{(18)}$. 
Quadro 1 - Principais alterações encontradas no respirador oral

\begin{tabular}{|c|c|}
\hline Crescimento & $\begin{array}{l}\text { - Aumento vertical do terço inferior } \\
\text { da face } \\
\text { - Arco maxilar estreito } \\
\text { - Palato em ogiva } \\
\text { - Ângulo goníaco obtuso } \\
\text { - Má-oclusão: mordida aberta, } \\
\text { dentes incisivos superiores em } \\
\text { protrusão e mordida cruzada } \\
\text { - Crescimento craniofacial vertical }\end{array}$ \\
\hline Laríngeas & $\begin{array}{l}\text { - Hióide mais baixo } \\
\text { - Alteração da musculatura } \\
\text { suprahioídea }\end{array}$ \\
\hline Muscular & $\begin{array}{l}\text { - Lábio superior e inferior } \\
\text { encurtados } \\
\text { - Maior atividade eletromiográfica } \\
\text { dos orbiculares da boca } \\
\text { - Hipofunção dos músculos } \\
\text { elevadores da mandíbula } \\
\text { - Alteração muscular e postural da } \\
\text { língua } \\
\text { - Mentual retraído }\end{array}$ \\
\hline Funções orais & $\begin{array}{l}\text { - } \text { Qualidade vocal hipo ou } \\
\text { - } \text { hipernasal e rouca } \\
\text { - } \text { Mala imprecisa } \\
\text { - Deglutição ineficiente atípica }\end{array}$ \\
\hline Face & $\begin{array}{ll}\text { - } & \text { Longa e estreita } \\
\text { - } & \text { Nariz pequeno e estreito } \\
\text { - } & \text { Cianose infra-orbitária } \\
\text { - } & \text { Falta de expressividade } \\
\text { - Incompetência labial } \\
\text { - } & \text { Lábios ressecados ou excesso } \\
& \text { de saliva em comissura labial }\end{array}$ \\
\hline
\end{tabular}

Quadro 2 - Seqüência de eventos relacionados à alteração de crescimento facial decorrente de obstrução nasal
$1^{\circ}$ Obstrução nasal
$2^{\circ}$ Postura de lábios separados
$3^{\circ}$ Respiração oral
$4^{\circ}$ Modificação do crescimento facial

A respiração oral é um dos sintomas mais freqüientes na infância $^{(19)}$ e grande parte da literatura ${ }^{(13,15,18-38)}$ a relaciona diretamente às alterações do crescimento facial, fala, distúrbios alimentares, alterações posturais, dificuldades escolares e doenças do sono, que interferem na qualidade de vida da criança $a^{(5)}$.

Pela variedade de causas e manifestações, muitos autores denominam "síndrome do respirador oral" às características encontradas nos indivíduos que utilizam a boca para respirar, sendo que tais manifestações desencadeiam inúmeras adaptações ${ }^{(37)}$ que, se não tratadas precoce e corretamente, irão trazer conseqüências por toda a vida. No Quadro 1, observam-se as principais alterações encontradas no indivíduo respirador oral e largamente citadas pela literatura.

O crescimento craniofacial é determinado por vários fatores, como herança genética e respiração oral crônica, a qual, quando se instala durante a fase de crescimento da criança, provocará alteraç̃oes morfológicas no complexo craniodentofacial. A obstrução nasal, portanto, pode estar associada à presença de doenças, sendo a rinite alérgica acompanhada ou não de hipertrofia de amígdala palatina ou faríngea a mais freqüente $\mathrm{e}^{(24)}$.

No Quadro 2, Vig ${ }^{(22)}$ propõe uma seqüência de eventos para explicar a alteração do crescimento facial vertical em conseqüência à obstrução nasal. Um distúrbio respiratório pode ser a chave determinante da abertura da boca, resultando no deslocamento mandibular ${ }^{(33)}$, encontrado com freqüência em indivíduos com tendência de tipo facial dolicofacial ${ }^{(31)}$.

Por outro lado, a respiração oral obriga o paciente a manter a boca aberta para suprir a deficiência de ar respirado. Com isso, o equilíbrio vestíbulo-lingual é removido, alterando o equilíbrio da musculatura facial ${ }^{(23)}$ e gerando uma deficiência funcional importante.

Pela falta de fluxo aéreo nasal, a pressão da língua no palato é reduzida, desviando a mandíbula para baixo e para trás em relação à base do crânio ${ }^{(25)}$, onde os músculos abaixadores da mandíbula exercem sobre ela uma tração muscular para trás, a cada inspiração. Devido a este abaixamento mandibular, os dentes superiores são privados de seu suporte muscular e pressão lateral. A partir desta relação instável entre forças musculares externas e internas sobre a boca, o músculo bucinador causa uma pressão no arco maxilar, resultando em estreitamento ${ }^{(21)}$, associando o comprometimento respiratório às deformidades dentofaciais ${ }^{(18)}$.

Com as alterações dos músculos elevadores da mandíbula, as funções orais, como mastigação e deglutição, estarão prejudicadas. Como a criança respira pela boca, ela se cansa e não come o suficiente ou, às vezes, come muito e rápido 
ou come várias vezes ao $\mathrm{dia}^{(17)}$, explicando a magreza ou a obesidade nas crianças respiradoras orais. Por outro lado, a presença da obstrução nasal altera o limiar de detecção do gosto salgado dos alimentos ${ }^{(39)}$, prejudicando o prazer da criança em se alimentar.

Já na deglutição, Bicalho et al ${ }^{(37)}$ enfatizam a prevalência da participação da musculatura perioral, com projeção anterior da língua e cabeça, deglutição ruidosa, interposição de lábio inferior e modificações relevantes na postura de repouso dos lábios.

Além das alterações posturais dos órgãos fonoarticulatórios, a obstrução nasal pode ser causadora ou mantenedora de cabeça mal posicionada em relação ao pescoço, com conseqüências para a coluna ${ }^{(13)}$. Ou seja, há estreita relação entre o modo de respirar, as posturas de cabeça e mandíbula e as atividades posturais dos músculos do pescoço, como o esternocleidomastóideo e o trapézio ${ }^{(32)}$, e a atividade eletromiográfica do músculo genioglosso ${ }^{(40)}$. A projeção anterior da cabeça causa também mudanças na tensão da língua, face (especialmente do músculo bucinador) e nos músculos supra e infrahioídeos, que contraem o músculo constritor da faringe, retificando o espaço oronasofaríngeo e facilitando a entrada de ar pela boca ${ }^{(41)}$.

Com o mesmo raciocínio, pode-se considerar os distúrbios do sono nas crianças respiradoras orais. Observam-se, em associação à respiração oral e à conseqüente redução do espaço da rinofaringe, sinais como baba noturna, roncos e distúrbios respiratórios decorrentes de alergia ${ }^{(29)}$ e, nos casos mais graves, a síndrome da apnéia e hipopnéia obstrutiva do sono, caracterizada por episódios repetitivos de obstrução das vias aéreas superiores durante o sono, usualmente associados à interrupção do mesmo e à queda da saturação da oxihemoglobina $^{(36)}$. A qualidade do sono também pode ser prejudicada na presença de rinite alérgica. Ferguson ${ }^{(30)}$ explica que vários sintomas, como espirro, prurido, rinorréia e congestão nasal, podem interferir no sono noturno e causar fadiga e sonolência no dia seguinte, reduzindo o aprendizado e a eficiência do trabalho, e diminuindo a qualidade de vida.

Há evidências de que a rinite alérgica aumenta o risco de síndrome da apnéia obstrutiva do sono na criança ${ }^{(42)}$ pelo aumento da resistência nasal, a qual já está prejudicada durante o sono ${ }^{(43)}$. A qualidade do sono e a da respiração, portanto, estão associadas. Tal fato explica a relação das alterações no sono com as dificuldades de atenção e concentração necessárias para a aprendizagem ${ }^{(17)} \mathrm{e}$ indica que crianças respiradoras orais apresentam maior risco de desenvolver dificuldade de aprendizagem $^{(44)}$ e transtornos de déficit de atenção/hiperatividade $^{(38,45)}$.

Além de todas as alterações citadas, a respiração oral pode acarretar consequiências mais graves como o Cor Pulmonale, cuja manifestação é a insuficiência cardíaca decorrente do grande aumento de resistência ao fluxo sangüíneo pulmonar ${ }^{(14)}$. Embora não haja estudos epidemiológicos quanto a sua incidência e prevalência - apenas relatos de casos isolados - tal complicação merece ser mencionada e estudada pela sua gravidade.

\section{Conclusão}

A asma e a rinite são doenças inter-relacionadas que apresentam, de maneira secundária, a obstrução nasal, a qual pode ser causadora e mantenedora de alterações orofaciais. O diagnóstico deve ser o mais precoce possível e o acompanhamento, multidisciplinar. Ou seja, o médico deverá pesquisar e tratar a obstrução nasal e/ou a asma; o ortodontista corrigirá as alterações dentárias após a eliminação da causa da respiração oral; e o fonoaudiólogo deverá reeducar e adaptar a respiração, adequando as funções orais e equilibrando a musculatura do sistema estomatognático. Ressalta-se, portanto, a unicidade entre a asma e a rinite pelo tratamento médico, com visão integrada de uma via respiratória única e suas conseqüências no crescimento e desenvolvimento de todo o sistema motor oral. 


\section{Referências bibliográficas}

1. Jessen M, Malm L. Definition, prevalence and development of nasal obstruction. Allergy 1997;52:S3-6.

2. Passalacqua G, Ciprandi G, Canonica GW. The nose-lung interaction in allergic rhinitis and asthma: united airways disease. Curr Opin Allergy Clin Immunol 2001;1:7-13.

3. Camargos PA, Rodrigues ME, Lasmar LM. Simultaneous treatment of asthma and allergic rhinitis. Pediatr Pulmonol 2004;38:186-92.

4. Annesi-Maesano I. Epidemiological evidence of the occurrence of rhinitis and sinusitis in asthmatics. Allergy 1999;54:S7-13.

5. Ibiapina CC, Sarinho LE, Cruz Filho AA, Camargos PA. Rinite, sinusite e asma: indissociáveis? J Bras Pneumol 2006;32:357-66.

6. Widdicombe J. Microvascular anatomy of the nose. Allergy 1997;52:S7-11.

7. Koh YY, Kim CK. The development of asthma in patients with allergic rhinitis. Curr Opin Allergy Clin Immunol 2003;3:159-64.

8. Braunstahl G, Overbeek SE, Kleinjan A, Prins JB, Hoogsteden HC, Fokkens W. Nasal allergen provocation induces adhesion molecule expression and tissue eosinophilia in upper and lower airways. J Allergy Clin Immunol 2001;107:469-76.

9. Camargos PA, Rodrigues ME, Sole D, Scheinmann P. Asma e rinite alérgica como expressão de uma única doença: um paradigma em construção. J Pediatr (Rio J) 2002;78:S123-8.

10. Togias A. Rhinitis and asthma: evidence for respiratory system integration. J Allergy Clin Immunol 2003;111:1171-83.

11. Kanani AS, Broder I, Greene JM, Tarlo SM. Correlation between nasal symptoms and asthma severity in patients with atopic and nonatopic asthma. Ann Allergy Asthma Immunol 2005;94:341-7.

12. Braunstahl GJ, Fokkens W. Nasal involvement in allergic asthma. Allergy 2003;58:1235-43.

13. Ferreira ML. A incidência de respiradores bucais em indivíduos com oclusão classe II. J Bras Ortodon Ortop Facial 1999;4:223-40.

14. Di Francesco RC. Respirador bucal: a visão do otorrinolaringologista. J Bras Ortodon Ortop Facial 1999;4:241-7.

15. Parolo AM, Bianchini EM. Pacientes portadores de respiração bucal: uma abordagem fonoaudiológica. Dent Press Ortodon Ortop Facial 2000;5:76-81.

16. Chadha TS, Birch S, Sackner MA. Oronasal distribution of ventilation during exercise in normal subjects and patients with asthma and rhinitis. Chest 1987;92:1037-41.

17. Bernardes FF. Respiração bucal: o que os pais sabem e respeito? Rev Cefac 1999;1:18-25.

18. Lessa FC, Enoki C, Feres MF, Valera FC, Lima WT, Matsumoto MA. Influência do padrão respiratório na morfologia craniofacial. Rev Bras Otorrinolaringol 2005;71:156-60.

19. Di Francesco RC, Passeroti G, Paulucci B, Miniti A. Respiração oral na criança: repercussões diferentes de acordo com o diagnóstico. Rev Bras Otorrinolaringol 2004;70:665-70.

20. Prates NS, Magnani MB, Valdrighi HC. Respiração bucal e problemas ortodônticos. Relação causa-efeito. Rev Paul Odontol 1997;19:14-6.

21. Tomé MC, Marchiori SC. Estudo eletromiográfico dos músculos orbiculares superior e inferior da boca em crianças respiradoras nasais e bucais durante o repouso com e sem contato labial. J Bras Ortodon Ortop Facial 1998;3:59-66.

22. Vig KW. Nasal obstruction and facial growth: the strength of evidence for clinical assumptions. Am J Orthod Dentofacial Orthop 1998;113:603-11.

23. Cintra CF, Castro FF, Cintra PP. As alterações oro-faciais apresentadas em pacientes respiradores bucais. Rev Bras Alergia Imunopatol 2000;23:78-83.
24. Motonaga SM, Berte LC, Anselmo-Lima WT. Respiração bucal: causas e alterações no sistema estomatognático. Rev Bras Otorrinolaringol 2000;66:373-9.

25. Faria PT, Ruellas AC, Matsumoto MA, Alselmo-Lima WT, Pereira FC. Dentofacial morphology of mouth breathing children. Braz Dent J 2002;13:129-32.

26. Ribeiro F, Bianconi CL, Mesquita MC, Assencio-Ferreira VJ. Respiração oral: alterações oclusais e hábitos orais. Rev Cefac 2002;4:187-90.

27. Rizzo C, Hauache S, Naspitz C, Pignatari S, Junqueira P, Hallinan M et al Characteristics of children with allergic rhinitis and chronic mouth breathing. J Allergy Clin Immunol 2002;109:S263.

28. Sabatoski CV, Maruo H, Camargo ES, Oliveira JH. Estudo comparativo de dimensões craniofaciais verticais e horizontais entre crianças respiradoras bucais e nasais. J Bras Ortodon Ortop Facial 2002;7:246-57.

29. Paulo CB, Conceição CA. Sintomatologia do respirador oral. Rev Cefac 2003;5:219-22.

30. Ferguson BJ. Influences of allergic rhinitis on sleep. Otolaryngol Head Neck Surg 2004;130:617-29.

31. Mattar SE, Anselmo-Lima WT, Valera FC, Matsumoto MA. Skeletal and occlusa characteristics in mouth-breathing pre-school children. J Clin Pediatr Dent 2004;28:315-8.

32. Ribeiro EC, Marchiori SC, Silva AM. Electromyographic muscle EMG activity in mouth and nasal breathing children. Cranio 2004;22:145-50.

33. Shikata N, Ueda HM, Kato M, Tabe H, Nagaoka K, Nakashima Y et al. Association between nasal respiratory obstruction and vertical mandibular position. J Oral Rehabil 2004;31:957-62.

34. Andrade FV, Andrade DV, Araújo AS, Ribeiro ACC, Deccax LDG, Nemr K Alterações estruturais de órgãos fonoarticulatórios e más oclusões dentárias em respiradores orais de 6 a 10 anos. Rev Cefac 2005;7:318-25.

35. Guilleminault C, Lee JH, Chan A. Pediatric obstructive sleep apnea syndrome. Arch Pediatr Adolesc Med 2005;159:775-85.

36. Salles C, Campos PSF, Andrade NA, Daltro C. Síndrome da apnéia e hipopnéia obstrutiva do sono: análise cefalométrica. Rev Bras Otorrinolaringol 2005;71:369-72.

37. Bicalho GP, Motta AR, Vicente LC. Avaliação da deglutição em crianças respiradoras orais. Rev Cefac 2006;8:50-5.

38. Vera CFD, Conde GE, Wajnsztejn R, Nemr K. Transtornos de aprendizagem e presença de respiração oral em indivíduos com diagnóstico de transtornos de déficit de atenção/hiperatividade (TDAH). Rev Cefac 2006;8:441-55.

39. Pellegrini G, Veleiro RVB, Gomes ICD. A percepção do gosto salgado em indivíduos com e sem obstrução nasal. Rev Cefac 2005;7:311-7.

40. Takahashi S, Ono T, Ishiwata Y, Kuroda T. Breathing modes, body positions, and suprahyoid muscle activity. J Orthod 2002;29:307-13.

41. Lima LC, Baraúna MA, Sologurem MJ, Canto RS, Gastaldi AC. Postural alterations in children with mouth breathing assessed by computerized biophotogrammetry. J Appl Oral Sci 2004;12:232-7.

42. Ng DK, Chan CH, Hwang GY, Chow PY, Kwok KL. A review of the roles of allergic rhinitis in childhood obstructive sleep apnea syndrome. Allergy Asthma Proc 2006;27:240-2

43. Pevernagie DA, De Meyer MM, Claeys S. Sleep, breathing and the nose Sleep Med Rev 2005;9:437-51.

44. Weber SA, Lima Neto AC, Ternes FJS, Montovani JC. Distúrbio de hiperatividade e déficit de atenção na síndrome de apnéia obstrutiva do sono: há melhora com tratamento cirúrgico? Rev Bras Otorrinolaringol 2006;72:124-9.

45. Wendel A, Albejante MFC, Coladeti AP, Assencio-Ferreira VJ. Relação causal entre respiração oral e dificuldades na aprendizagem. Rev Cefac 2002;4:137-40. 